\title{
Anatomical rules for the plastic repair of a diseased mitral valve
}

\author{
R. W. M . F R A T E R \\ From the Departments of Surgery and Cardiothoracic Surgery, Medical School, University of Cape Town, \\ South Africa, and the C.S.I.R. Cardiovascular-Pulmonary Research Group ${ }^{1}$
}

Although total replacement of the mitral valve is becoming commonplace, there still exists a large group of cases for which plastic techniques, using the patient's own tissues, are feasible and perhaps preferable. It appears from most published accounts of open surgery for the diseased mitral valve that the attainment of a valve with completely normal function post-operatively is unusual (Anderson, Cobb, Bruce, and Merendino, 1962 ; Clowes, Neville, Sancetta, Traks, Lim, Barwinsky, and Del Guercio, 1962 ; Kay, Egerton, and Zubiate, 1961 ; Morris, Sloan, Wilson, and Brandt, 1962). Various defects leading to insufficiency and many techniques of repair have been described (Brock, 1952 ; Barnard, McKenzie, and Schrire, 1961; Levy and Edwards, 1962). However, the application of the techniques to the repair of a particular diseased valve seems to be very much an art. Specific and detailed information is not presented of how to analyse a valve deformity and how to estimate the changes in its dimensions and form that are needed to correct it. In this paper an attempt is made to formulate 'rules' for doing this.

\section{MATERIAL AND METHODS}

Normal canine and normal and abnormal human hearts were studied by dissection and by observation when being made to beat in an external pulse duplicator. Slow motion films were taken of some specimens and repeatedly restudied. From the observations on normal hearts an analysis of the factors necessary for normal valve function was made. The accuracy of this analysis was tested in vitro and in vivo by using it as the basis for the design and tailoring of prosthetic cusps and chordae for the replacement of parts of normal mitral cusps and chordae. In vitro the function of the modified valve was tested by the use of the

\footnotetext{
${ }^{1}$ Part of this work was performed at the Mayo Foundation, Rochester, Minnesota, and was aided by the Minnesota Heart Association Funds.
}

external pulse duplicator. In the live experiments, valve function was carefully assessed by directo observation with the ventricle filled with blood, bypalpation with the heart beating, and bycs simultaneous left ventricular and left atriato pressure measurements. If the animals died post-跨 operatively, the external pulse duplicator was usedo again. A further test was provided by the use of ${ }^{-}$ these design rules in the assessment and plastice repair of cases of mitral insufficiency.

\section{BASIC ANATOMICAL FACTS}

The basic anatomical facts that determine competent function of the mitral valve are very simple. They are:

1. The valve has two main cusps, the anterior being slightly wider and deeper than the posterior. Although the levels of their bases usually differ, their free edges are at the same level in the ventricular cavity relative to its long axis. The sum of the lengths of these main cusps is always more $x$ than the diameter of the relaxed ring and clearly을 more than that of the contracted ring (Fig. 1a).

2. The chordae tendineae rise at different levelsô from the papillary muscles and are inserted at different levels on the cusps. Yet all are always윽 at the same tension. The corollary to this is that the relationship of the free edges of the main cusps in the relaxed state will be maintained during systole. Measurements of chordae do not reveal any formula for length. For instance, chordae to the anterior cusp arising from the antero-lateral ${ }^{N}$ papillary muscle may differ in length from those from the postero-medial papillary muscle or fromo those to an opposite part of the posterior cusp. However, the distance from the free edge of a cusp? to a point of distal support is always less than the distance from the valve ring to that point (Fig. 1b). $\frac{\text { ( }}{\Phi}$ A greater length than this results immediately in $\stackrel{\mathbb{Q}}{\stackrel{9}{ }}$ failure of the main cusps to meet (Fig. 2a). So $\stackrel{\mathbb{D}}{\mathscr{D}}$ long as these conditions are met, the chordae may으 

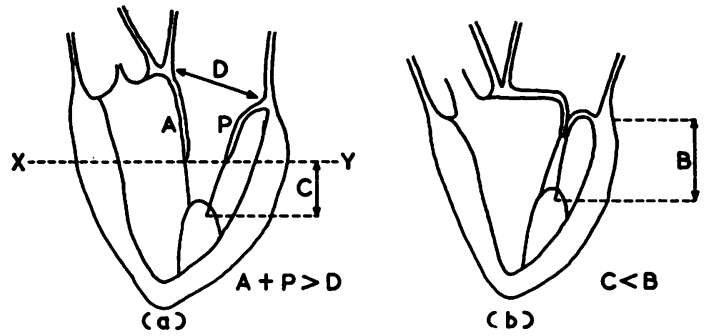

FIG. 1. (a) Cross-section through left ventricle bisecting aortic and mitral valves. Diastole. $A$, length of anterior cusp of mitral valve; $P$, length of posterior cusp of mitral valve; $D$, diameter of the mitral valve ring; $C$, distance from free edge of valve to point of distal attachment; $X Y$, plane of the level to which the main cusps descend. (b) The same. Systole. B, distance from point of distal attachment to atrioventricular ring.

vary greatly in length and yet allow competent function. The length and position of the papillary muscles largely determine their length. They may be quite short so long as the mobility of the cusp they are attached to is not affected.

3. The two main cusps are on opposite sides of the ring. They are joined by the shallow but distinct and definite commissural tissue. The continuity of tissue around the valve ring is never interrupted (Fig. 3a and b). Because the anterior cusp is rather larger than the posterior, it makes contact with more of the commissural tissue in closure than does the posterior. This separation of the main cusps is essential to avoid stenosis. The bridging of the space between them is essential to prevent incompetence.

4. In the healthy valve a striking feature is the pliability and flexibility of the cusps and chordae. This property is important for optimal function
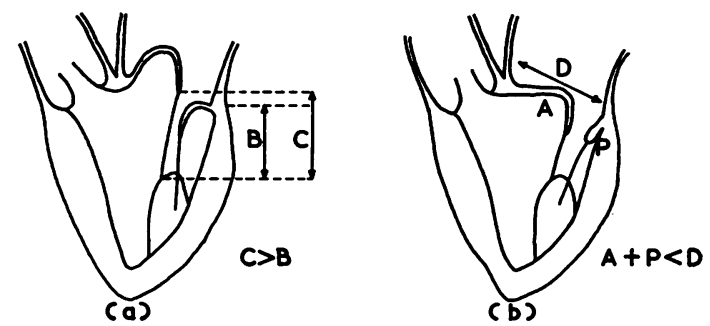

FIG. 2. (a) Left ventricular cross-section. Mitral insufficiency due to excessive length of anterior cusp chordae tendineae. $C$, distance from point of distal attachment of chordae tendineae to the free edge of the valve; $B$, distance from point of distal attachment to atrio-ventricular ring. (b) Mitral insufficiency due to shortening of posterior cusp. $A$, length of anterior cusp; $P$, length of posterior cusp; $D$, diameter of atrio-ventricular ring.

of the mitral valve. In particular, the perfect apposition of cusps in closing is very dependent on pliability.

\section{DESIGN RULES}

These are also simple, being merely the anatomical facts stated differently.

1. When a cusp is being lengthened or replaced, its length must be such that it hangs to the same depth into the ventricle as the remaining normal cusp. The sum of the lengths of the two cusps should be more than the diameter of the relaxed ring (Figs. $1 \mathrm{a}$ and $2 \mathrm{~b}$ ).

2. When new chordae are made with the heart relaxed and the cusp lengths correct, their length should be such that their tension is the same as that of remaining normal chordae. In addition, their lengths should be less than the distance from their papillary attachments to the ring (Fig. 1b
FIG. 3. (a) View of the mitral valve from the left atrium. (b) Mitral valve dissected and laid out flat. A, anterior cusp; $P$, posterior cusp; $C$, commissural tissue.

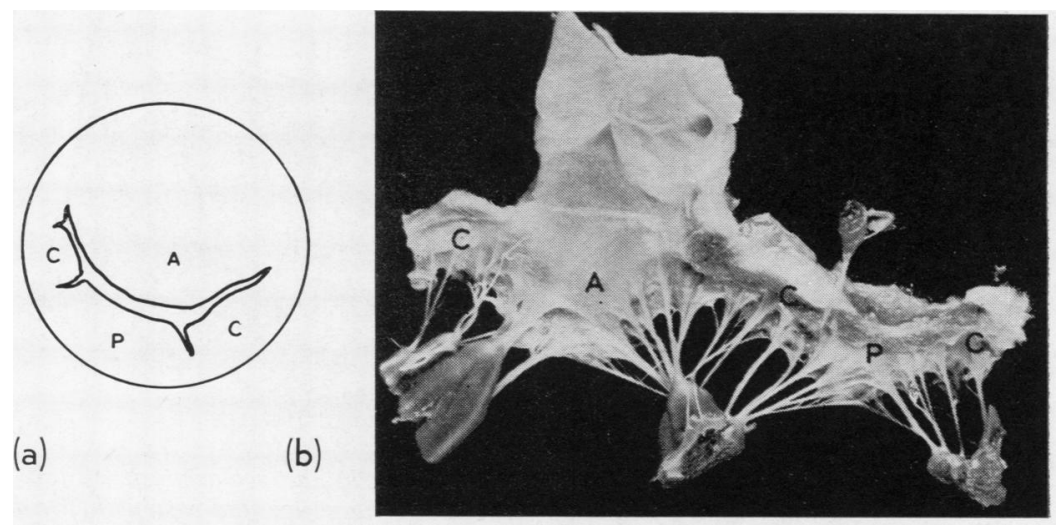


and Fig. 2a). For the special circumstance of the valve repaired by the insertion of an immobile baffle, the remaining cusp length must exceed the orifice diameter, and the chordae must be shorter than the papillary muscle to baffle measurement (Fig. 4).

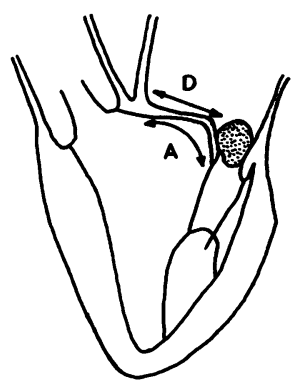

FIG. 4. Left ventricular cross-section. The shortening of the posterior cusp has been corrected by the insertion of an immobile 'ivalon' baffle. $A$, length of anterior cusp; $D$, new diameter of the ring after insertion of baffle. With competence $A>D$.
3. The continuity of valve tissue around the $\frac{\bar{C}}{0}$ ring must always be maintained (Fig. $3 a$ and b). $\frac{\bar{\rho}}{\sigma}$

4. Flexibility and pliability must be preserved $\stackrel{\odot}{\odot}$ for optimal results. Flexibility ideally should be such that gravity determines the position of a cusp. क For instance, it should fall into the ventricular $\stackrel{\circ}{\circ}$ cavity by its own weight in the opened heart. $\overrightarrow{\vec{\omega}}$ When a part of a cusp is picked up the surrounding valve tissue should fall away around $\overrightarrow{\vec{x}}$ it.

5. If the tissues have the requisite flexibility, the $\overrightarrow{\overrightarrow{0}}$ avoidance of stenosis becomes a matter merely of ensuring a minimum orifice area.

\section{EXPERIMENTAL APPLICATION OF THE RULES}

The rules were tested experimentally by the $\mathbb{D}$ replacement of parts of the canine mitral valve $\mathbb{\mathbb { D }}$ by pericardial cusps and chordal sheets. Experi- $\frac{\square}{0}$ ments were first performed in vitro using an $\frac{\mathbb{C}}{-}$ external pulse duplicator to simulate cardiac $\vec{\varphi}$ action. The posterior mitral cusp, the antero- $\$$ lateral and postero-medial commissural tissue, and

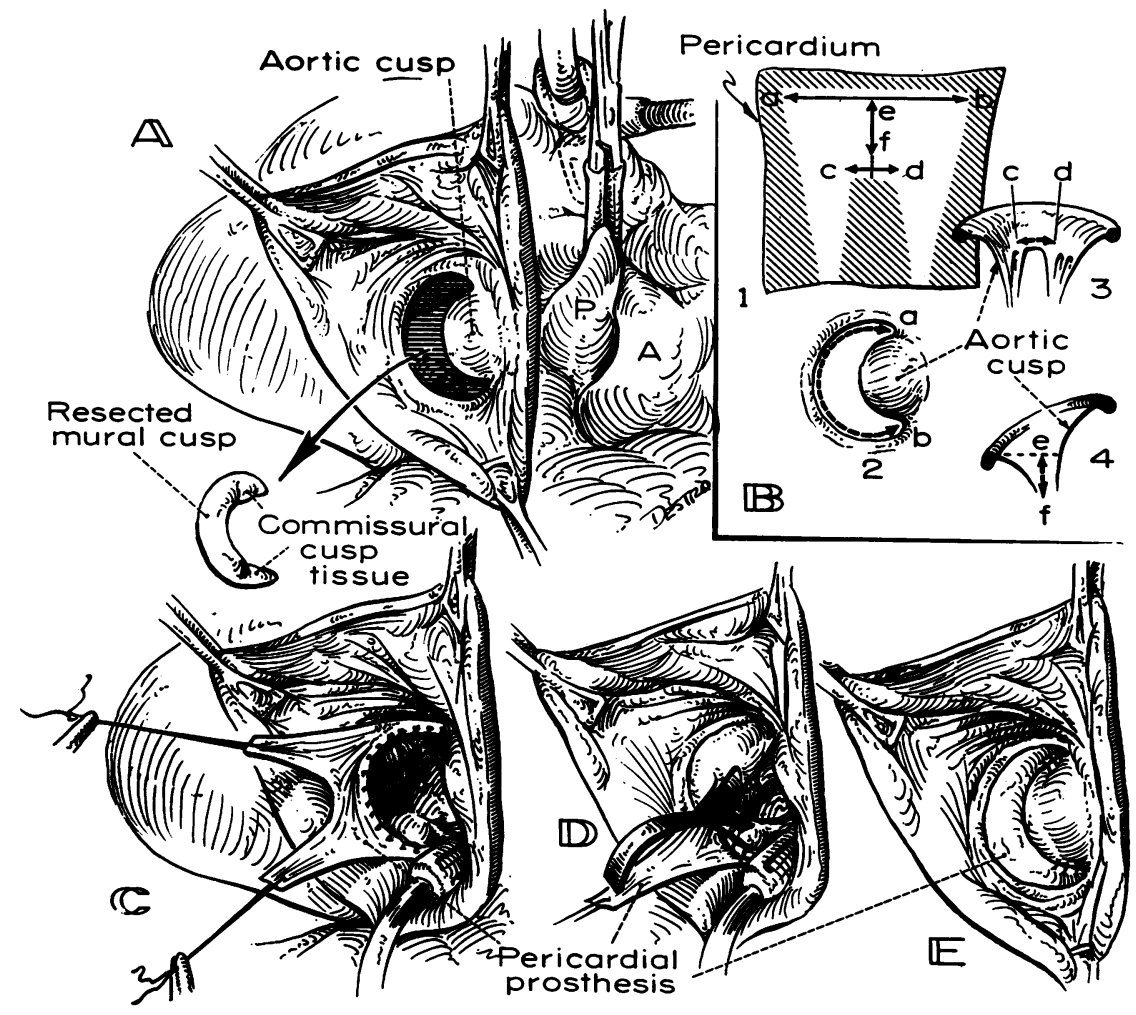

FIG. 5. Drawing of the stages involved in the replacement of the posterior cusp, com missural tissue, and chordae tendineae by a pericardial cusp with chordal sheets. 
their chordae tendineae were replaced by pericardial cusps and chordal sheets. In 15 experiments insufficiency occurred once when the length of one of the chordal sheets was made too long. In vivo, cardiopulmonary bypass was used to perform the following replacements: posterior cusp and chordae (once-no insufficiency); posterior cusp, commissural tissue, and chordae (13 times-gross insufficiency twice, mild localized insufficiency twice) (Fig. 5); anterior cusp and chordae (eight times-mild localized insufficiency once) ; chordae to one side of anterior cusp (twice -mild insufficiency both times); whole mitral valve (once-no insufficiency); anterior cusp in combination with a posterior baffle (once-no insufficiency). These experiments were all performed by the author. When insufficiency occurred it could always be explained on the basis of a failure to apply the rules correctly. In one of the two examples of gross insufficiency, the length of the prosthetic cusp had been made far too short; in the other, one of the chordal sheets was too tight.

Both the successful and the unsuccessful experiments bear out the validity of the 'rules'.

\section{CLINICAL APPLICATION}

To any surgeon familiar with the open repair of mitral valve disease it will be clear that the various types of disorder found can be related to a deficiency of one or more of the basic factors determining good valve function that have been enumerated in this article. The author has found that a formal and systematic appraisal of each of the factors in turn makes possible a rapid and accurate assessment of the cause or causes of the valve dysfunction. Many surgeons no doubt arrive at similarly accurate assessments by 'eye' and by virtue of experience: for the tyro, having a plan to follow can simplify this task.

The following simple plan is suggested. Information must be obtained regarding (1) the diameter of the ring; the lengths of the major cusps: (2) the lengths of the chordae tendineae or groups thereof in relation to the distance between their papillary muscle origin and the ring; the levels in the ventricle at which the chordae hold the edges of the major cusps: (3) the integrity and depth of the commissural tissue: and (4) the pliability and flexibility of all cusp tissue and chordae.

A small selection of cases is presented to illustrate the application of this plan. Only the operative findings, the technique of repair, and the early post-operative results are discussed.
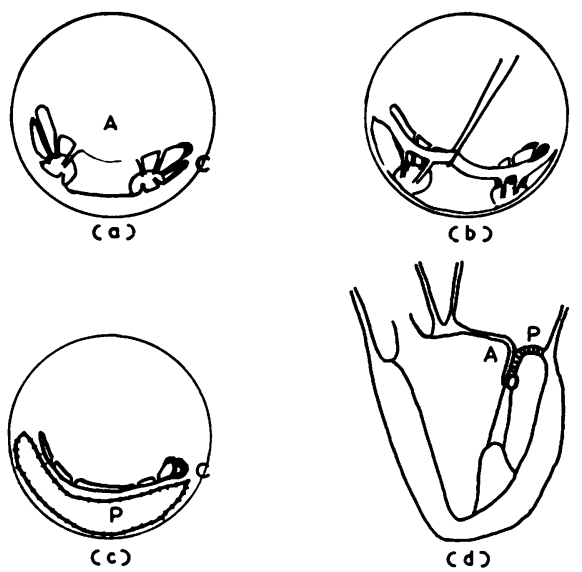

FIG. 6. Drawing of the findings in case 1. (a) Left atrial view. The anterior cusp $(A)$ is slightly shortened. The posterior cusp and the commissural tissue are grossly shortened. The postero-medial commissural tissue $(C)$ is thick and immobile. (b) The shortened tissue with its chordae tendineae has been separated from the atrioventricular ring. (c) A pericardial autograft $(P)$ has been stitched to ring and cusp. The anterior cusp now meets the posterior cusp and commissural tissue except at $C$ where the rigidity of the tissue prevents apposition despite the lengthening produced by the insert. (d) Left ventricular cross-section. Compare with 2 (b).

CASE 1 No effective apposition of any part of the cusps took place (Fig. 6). The ring diameter (heart beating) was $5 \mathrm{~cm}$. The anterior cusp was slightly shortened. Its length was $3 \mathrm{~cm}$. The posterior cusp was contracted to $0.5 \mathrm{~cm}$. The commissural tissue was shortened, especially postero-medially. The chordae of the posterior cusp were fused medially with the ventricular wall. They were dissected free, and the posterior cusp was separated from the ring. It was then evident that the lengths of the chordae to both main cusps were such that the free edges were at the same level and did not project above the plane of the atrioventricular ring. The commissural tissue medially was very short, but, more important than this, it had lost all its pliability or flexibility. The flexibility of the free edge of the posterior cusp, the anterior cusp, and the chordae was undoubtedly less than normal but was still such that its shape and position were 'gravity determined'. The posterior cusp and commissural tissue were lengthened by an insert of autogenous pericardium measuring $5 \times 3 \mathrm{~cm}$. This corrected all the insufficiency except that at the postero-medial commissure. Here the rigidity of the tissues prevented apposition with the anterior cusp. Although there was a good drop in the left atrial pressure post-operatively, and although there has been excellent clinical improvement, a grade 2 (basis 6) murmur of mitral insufficiency has been audible since the day of surgery. 
CASE 2 A closed commissurotomy had been done one year before with the production of severe traumatic mitral insufficiency. The ring was $4.5 \mathrm{~cm}$. in diameter. The anterior cusp was $3.5 \mathrm{~cm}$. long and the posterior cusp was $0.5 \mathrm{~cm}$. The chordae to the anterior cusp and the antero-lateral commissural tissue were of normal length. The posterior cusp chordae were obliterated by scar tissue. The antero-lateral commissural tissue was intact and of normal length, but where it joined the anterior cusp there was a tear extending right out to the ring. The postero-medial commissural tissue was fused with the adjacent anterior cusp, and the whole area was densely infiltrated with calcium. The anterior cusp laterally was near normal in flexibility, but its medial third was immobilized by calcium. The antero-lateral commissural tissue also had normal pliability but the whole of the rest of the valve had lost all flexibility (Fig. 7a).
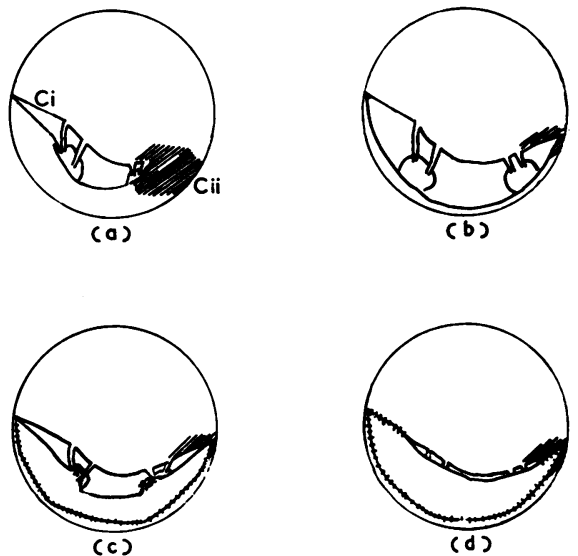

FIG. 7. (a) The tear out to the atrio-ventricular ring is at $C i$. The hatched area at $C$ ii represents calcification. (b) Appearance after excision of the diseased posterior cusp and commissural tissue. (c) The pericardial cusp and its chordal sheets have been stitched to the atrio-ventricular ring and the papillary muscles. (d) The commissural suture lines have been completed.

In order to cure the insufficiency, the tear out to the wall had to be at least partially sewn up. This would have had the effect of reducing the orifice diameter to $2 \mathrm{~cm}$. or less with mobile tissues on only one side of it. The lack of flexibility of the remaining tissue precluded their use in plastic procedures. The only alternative to complete replacement of the whole valve was replacement of the rigid tissue with a fully flexible prosthetic cusp and chordae. The posterior cusp and the medial commissural tissue and their chordae tendineae were excised. The medial part of the anterior cusp was debrided of calcium until a measure of flexibility had been returned to it. The excised tissue was then replaced by a pericardial cusp with chordal sheets tailored so that cusp and chordal lengths were correct according to the 'rules'. Care was taken to suture the graft to the adjacent $\vec{\Phi}$ natural tissue at each end so as to leave no gaps in the continuity of cusp tissue around the ring (Fig. 7). ڤ Aortic insufficiency was present in this patient, and $\vec{\circ}$ while still on perfusion it was shown that no blood leaked back from the ventricle into the atrium. The flexibility of the prosthetic cusp allowed adequate diastolic opening. Although one month after surgery a mitral systolic murmur recurred, the immediate result of the procedure was a complete correction of the insufficiency and stenosis.

CASE 3 In this patient the free edge of all the cusp $\infty$ tissue had become fused and shortened (Fig. 8). 을 Instead of the cusps being able to vary in position $\rightarrow$ from ballooned into the atrium to hanging loosely $\mathcal{N}$ down into the ventricle, they formed a tightly $\frac{\mathbb{D}}{0}$ stretched membrane between a fixed central orifice $\mathbb{D}$ and the valve ring. Both anterior and posterior cusps $\vec{\sigma}$ were shortened. The commissural tissue, while of $\mathbb{\Phi}$ normal depth, had lost all length along its free edge, $\vec{\varphi}$ and thus could no longer perform its functions of allowing the main cusps to open freely in diastole and filling the gap between them in systole. All the chordae were of normal length but crowded closer
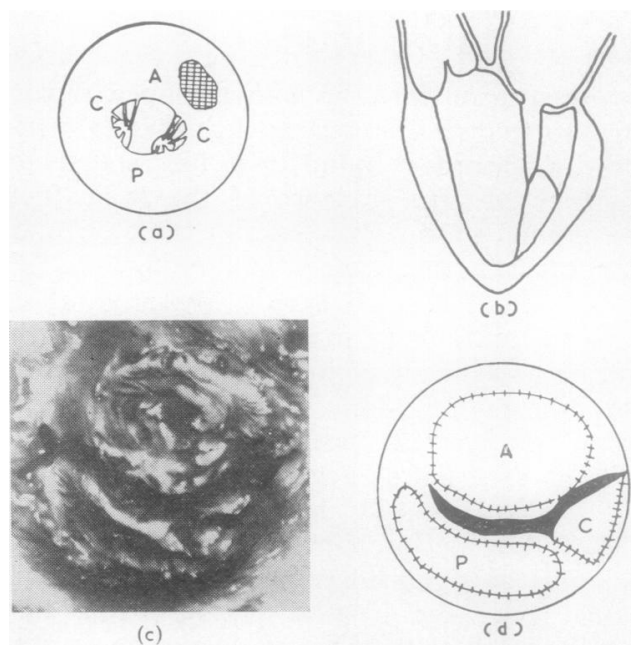

FIG. 8. Case 3. (a) and (b) The free edge of the leaflet tissue has contracted, producing a diaphragm stretched across the atrio-ventricular region. The valve orifice is fixed. Despite the immobility of the cusps the tissues are not thickened peripherally. $A$, anterior cusp; $P$, posterior cusp; $C$, commissural tissue. The cross-hatched area represents a plaque of calcium. (c) and $(d)$ Photograph and $T$ drawing of the post-mortem appearance of the repaired valve. $A$, pericardial autograft in anterior cusp; $P$, pericardial autograft in posterior cusp; $C$, commissural cusp and chordal sheet. The commissural suture line between $A$ and $C$ has given way. 
together than usual because of the shrinkage of the free edge of the valve. The flexibility of the chordae and of the cusp tissue at ring level was normal. The thickening of the contracted free edge of the valve produced some but not excessive diminution in pliability of this part of the valve. Pliable autografts of pericardium were inserted between the ring and the free edges of the main cusps. This cured the incompetence but, because of the contraction of the free edge of the commissural tissue, the main cusps could not open widely in diastole, and the valve remained stenotic. The main cusps were therefore separated at the postero-medial commissure, and a pericardial cusp and chordal sheet were inserted between the main cusps, being sutured to each of them, to the ring, and to the papillary muscle. The valve was then relieved of its stenosis and retained its competence.

There were no murmurs for the first two weeks after surgery, and the patient was making a dramatic recovery from a state of continuous cardiac failure and complete invalidism. At this stage he developed bacterial endocarditis due to a Staphylococcus albus. The murmur of mitral insufficiency gradually returned and cardiac failure recurred. The patient eventually died two months after surgery in gross congestive cardiac failure with his endocarditis uncured. At necropsy the suture line between the anterior cusp and the prosthetic commissural cusp had given way, perhaps as a result of the bacterial infection. The insufficiency had been due to this one defect.

CASE 4 In this patient the ring diameter was $4 \mathrm{~cm}$. The anterior cusp was considerably shortened, being only $2.5 \mathrm{~cm}$. long. The length of the posterior cusp was $0.5 \mathrm{~cm}$. The antero-lateral commissural tissue was only slightly shortened, but the postero-medial commissural tissue was grossly diseased, being very short, thick, and immobile. The mobility of the other cusp tissue was satisfactory. The chordae tendineae to the medial side of the anterior cusp were excessively long, longer in fact than the distance from their point of papillary origin to the plane of the atrio-ventricular ring. The remaining chordae were normal in length. Three defects needed correction: the posterior cusp needed lengthening; the rigidity, shortening, and immobility of the postero-medial commissural tissue had to be dealt with; and the medial chordae to the anterior cusp had to be shortened. Annuloplasty stitches had been used in other patients to combat the presence of elongated chordae tendineae or rigid, shortened commissural tissue, but it was felt that the results had been poor, and an alternative technique was tried. The posterior cusp and all the commissural tissue were separated from the atrio-ventricular ring. The thick and stiff postero-medial commissural tissue was then stitched to the adjacent anterior cusp edge by means of heavy silk mattress sutures buttressed by compressed ivalon. A patch of autogenous pericardium was then inserted between the freed posterior cusp and commissural tissue and the ring. The patch increased the length of the posterior cusp to $3 \mathrm{~cm}$.,

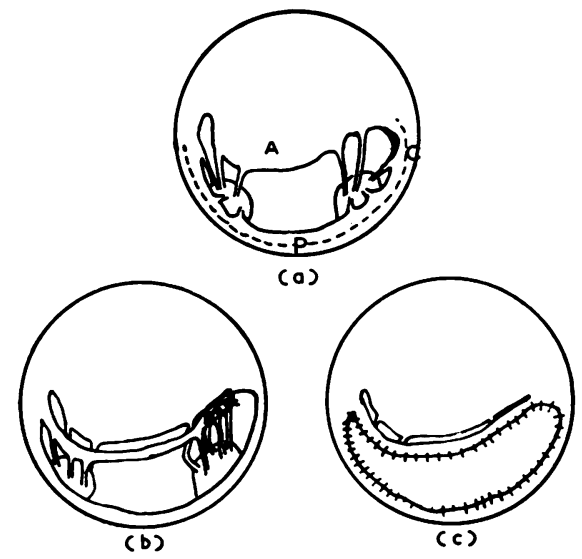

FIG. 9. Case 4. (a) A, grossly shortened anterior cusp with excessively long postero-medial chordae tendineae; $P$, grossly shortened posterior cusp; $C$, thickened ridged postero-medial commissural tissue. The dotted line is the line of incision between the cusp and atrio-ventricular ring. (b) The ridged and thickened commissural tissue has been stitched to the opposing part of the anterior cusp. (c) The pericardial insert is in place. The potentially troublesome commissural area has been obliterated, and the anterior cusp is supported by chordae tendineae of the proper length.

and at the posterior commissural area it was cut wide and square. By these manœuvres the rigidity of the commissural tissue could no longer prevent apposition with the anterior cusp since the heavy silk mattress sutures ensured this permanently. Furthermore, the anterior cusp was now supported by the commissural tissue chordae tendineae which were normal in length. Finally, the provision of a wide pericardial insert at this area helped to take the strain of the mattress sutures. Although there was some reduction in potential orifice this was not significant. The insufficiency was completely corrected and the patient is free of murmurs.

\section{DISCUSSION}

These four cases have been selected from a larger group to illustrate the clinical use of certain anatomical and functional concepts in plastic repair of the mitral valve. No particular brief is presented for the techniques used in these cases. In fact they have been selected as much to show the validity of the 'rules' in cases that have failed surgically as in those that have succeeded. The assessment of cusp length in relation to ring diameter, chordal length, the integrity of the commissural tissue, and the pliability of all parts of the valve can be a valuable prelude to the use of any technique of plastic repair. 
The question of cusp length comes up in all the four illustrative cases. Chordal length was of particular importance in case 4 . The importance of the integrity of the commissural tissue is emphasized in cases 2 and 3. Loss of this is most often seen after traumatic incompetence. The ill effects of loss of pliability are illustrated in cases 1 (commissural tissue), 2 (posterior cusp and postero-medial commissural tissue), and 4 (postero-medial commissural tissue). Mere shortening does not necessarily imply excessive loss of pliability. At the commissural areas, however, shortening and loss of pliability generally go together and are a frequent source of trouble with all kinds of plastic repair. Too great a loss of pliability is the major factor that makes total valve replacement necessary. When more than half of the cusp tissue has lost pliability, total valve replacement becomes mandatory.

\section{SUMMARY}

Simple anatomical rules governing the function of the mitral valve are presented. From them a scheme to be followed in assessing the nature of the valve defect during open plastic repair of the mitral valve has been derived.

Experience with the application of these 'rules' in the animal laboratory is reported. Four cases $\varrho$ are presented to illustrate the clinical application के of these concepts.

Thanks are due to Dr. J. G. Burger for permission to publish the cases. This work was supported by the J. S. Marais Surgical Research Grant.

\section{REFERENCES}

Andeison, A. M., Cobb, L. A., Bruce, R. A., and Merendino, K. A. 穴 (1962). Evaluation of mitral annuloplasty for mitral regurgi-
tation: clinical and hemodynamic status four to forty-one tation: clinical and hemodynamic status four to forty-one $\subseteq$
months after surgery. Circulation, 26, 26. Barnard, C. N., McKenzie, M. B., and Schrire, V. (1961). A surgical $\rightarrow$ approach to mitral insufficiency. Brit. J. Surg., 48, 655.

Brock, R. C. (1952). The surgical and pathological anatomy of the (D mitral valve. Brit. Heart J., 14, 489.

Clowes, G. H. A., Neville, W. E., Sancetta, S. M., Traks, E., Lim, K., Barwinsky, J., and Del Guercio, L. R. M. (1962). Results of open surgical correction of mitral valvular insufficiency and $\bar{\partial}$ description of technique for approach from left side. Surgery, 51, 迥 138.

Kay, J. H., Egerton, W. S., and Zubiate, P. (1961). The surgical treatment of mitral insufficiency and combined mitral stenosis and insufficiency with use of the heart-lung machine. Ibid., 50, 67. O

Levy, M. J., and Edwards, J. E. (1962). Anatomy of mitral insufficiency. Progr. cardiovasc. Dis., 5, 119.

Morris, J. D., Sloan, H., Wilson, W. S., and Brandt, R. L. (1962). O An appraisal of clinical results in open mitral valvuloplasty. J. thorac. cardiovasc. Surg., 43, 17. 